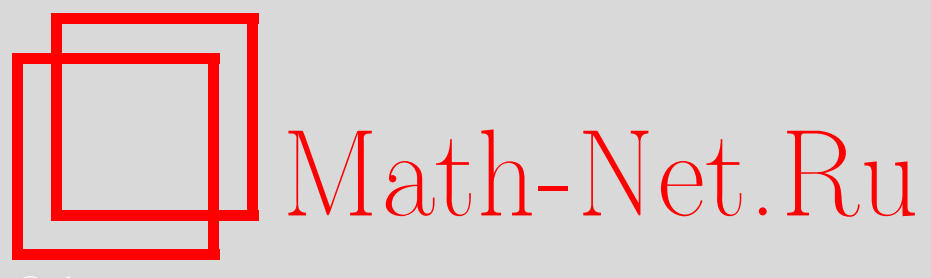

С. А. Степин, А. Г. Тарасов, Асимптотическое распределение резонансов для одномерного оператора Шрёдингера с финитным потенциалом, Матем. сб., 2007, том 198, номер 12, 87-104

DOI: https://doi.org/10.4213/sm3846

Использование Общероссийского математического портала Math-Net.Ru подразумевает, что вы прочитали и согласны с пользовательским соглашением http://www . mathnet.ru/rus/agreement

Параметры загрузки:

IP : 54.174 .149 .18

26 апреля 2023 г., 18:11:33

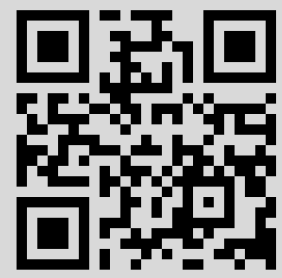




\author{
С. А. Степин, А. Г. Тарасов
}

\title{
Асимптотическое распределение резонансов для одномерного оператора Шрёдингера с финитным потенциалом
}

В работе изучается асимптотическое распределение резонансов, т.е. полюсов аналитического продолжения ядра резольвенты, оператора Шрёдингера. В предположении финитности потенциала установлено, что спектр резонансов состоит из двух серий, расположенных вблизи логарифмик, параметры которых определяются длиной носителя потенциала и порядками нулей на его концах. Основная теорема дополняет и усиливает известные результаты, а именно, позволяет рассматривать комплексные потенциалы с нулями произвольных (не обязательно целых) порядков касания на границе носителя и для таких потенциалов выписывать асимптотику с квалифицированной оценкой остатка.

Библиография: 11 названий.

\section{$\S 1$. Введение}

В работе рассматривается задача на собственные значения

$$
\begin{gathered}
-y^{\prime \prime}(x)+q(x) y(x)=k^{2} y(x), \\
y^{\prime}(a)-i k y(a)=0, \quad y^{\prime}(b)+i k y(b)=0,
\end{gathered}
$$

где спектральный параметр $k$ входит и в уравнение (1), и в граничные условия (2). Предполагается, что комплекснозначная функция $q(x)$ в точках $a$ и $b$ имеет нули порядков $\alpha$ и $\beta$ соответственно:

$$
q(x)=(x-a)^{\alpha} q_{0}(x)(b-x)^{\beta}, \quad q_{0}(a) q_{0}(b) \neq 0 .
$$

Под спектром задачи (1)-(2) понимается множество ее собственных значений.

Если продолжить $q(x)$ нулем за пределы отрезка $[a, b]$, то выполнение условий (2) означает, что соответствующая собственному значению $k$ собственная функция $y(x)$ допускает продолжение

$$
y(x)= \begin{cases}c_{1} e^{i k x}, & x \leqslant a, \\ c_{2} e^{-i k x}, & x \geqslant b,\end{cases}
$$

оставаясь при этом решением уравнения (1). Таким образом, если $k$ - собственное значение задачи $(1)-(2)$ и $\operatorname{Im} k<0$, то $y \in L_{2}(\mathbb{R})$ и, стало быть, $E=k^{2}-$

Работа выполнена в рамках Программы поддержки ведущих научных школ РФ (грант № НШ-4564.2006.1), а также поддержана Российским фондом фундаментальных исследований (грант № 05-01-00989).

(C) С. А. Степин, А. Г. ТАрасов, 2007 
собственное значение соответствующего оператора Шрёдингера с финитным потенциалом $q(x)$. Если же $\operatorname{Im} k>0$, то $E=k^{2}$ является резонансом, т.е. полюсом аналитического продолжения ядра резольвенты (функции Грина) указанного оператора. В случае $\operatorname{Im} k=0$ точка $E=k^{2}$ принадлежит непрерывному спектру и называется спектральной особенностъю (сингулярностъю) рассматриваемого оператора Шрёдингера.

Вопросам локализации и распределения резонансов в контексте задачи рассеяния уделялось внимание в ряде публикаций физиков и математиков, среди которых отметим монографии [1]-[4] и обзоры [5], [6]. В настоящей работе показано, что в нижней полуплоскости $\mathbb{C}_{-}=\{\operatorname{Im} k \leqslant 0\}$ имеется лишь конечное число точек спектра задачи (1)-(2), а в верхней полуплоскости $\mathbb{C}_{+}=\{\operatorname{Im} k \geqslant 0\}$ расположено счетное множество собственных значений и установлен асимптотический закон их распределения. Основным результатом данной работы является следующая

Teорема. Пусть $q_{0} \in C^{m}[a, b], m \geqslant \max \{\alpha, \beta\}+2$. Тогда в полуплоскосmи $\mathbb{C}_{+}$спектр задачи (1)-(2) состоит из двух серий собственных значений $\left\{k_{n}^{ \pm}\right\}$с асимптотиками

$$
\begin{aligned}
k_{n}^{ \pm}= \pm & \frac{\pi n}{b-a}+\frac{i \gamma \ln n}{2(b-a)}-\frac{i \ln C}{2(b-a)} \pm \frac{\pi \gamma}{4(b-a)} \\
& +\frac{i \gamma}{2(b-a)} \ln \left(\frac{2 \pi}{b-a}\right)+O\left(\frac{\ln n}{n}\right), \quad n \rightarrow+\infty
\end{aligned}
$$

где $C=\Gamma(\alpha+1) \Gamma(\beta+1) q_{0}(a) q_{0}(b)(b-a)^{\alpha+\beta}, \gamma=\alpha+\beta+4$.

Задача (1)-(2) в случае целых $\alpha$ и $\beta$ исследована в статье [7], где в предположении $m>\max \{\alpha, \beta\}$ вычислены собственные значения $k_{n}^{ \pm}$с точностью $o(1)$ при $n \rightarrow \infty$. Сформулированная выше теорема дополняет (и в определенном смысле усиливает) основной результат работы [7], а именно, она позволяет рассматривать комплексные потенциалы $q(x)$ с нулями произвольных, не обязательно целых, порядков касания в точках $a$ и $b$ и для таких потенциалов выписывать асимптотику собственных значений задачи (1)-(2) с квалифицированной оценкой остатка. В [8] рассмотрена задача Редже, отличающаяся от (1)-(2) заменой первого из краевых условий $(2)$ условием $y(a)=0$, и для нее изучено асимптотическое распределение точек спектра. Метод получения асимптотических формул (3) родствен подходу, используемому в [8], и существенно отличается от метода работы [7].

Известно (см., например, [9]), что спектр задачи (1)-(2) совпадает с нулями функции

$$
\Delta(k)=\left|\begin{array}{ll}
y_{1}^{\prime}(a, k)-i k y_{1}(a, k) & y_{2}^{\prime}(a, k)-i k y_{2}(a, k) \\
y_{1}^{\prime}(b, k)+i k y_{1}(b, k) & y_{2}^{\prime}(b, k)+i k y_{2}(b, k)
\end{array}\right|
$$

- так называемого характеристического определителя, который строится по фундаментальной системе решений (ФСР) $\left\{y_{1}(x, k), y_{2}(x, k)\right\}$ уравнения (1). В ходе доказательства основной теоремы (см. §5) в качестве ФСР уравнения (1) выбирается пара $y_{1}(x, k)=e^{-i k x} v_{1}(x, k)$ и $y_{2}(x, k)=e^{i k x} v_{2}(x, k)$, где $v_{1}(x, k)$ и 
$v_{2}(x, k)$ - решения интегральных уравнений

$$
\begin{aligned}
& v_{1}(x, k)=1+\frac{i}{2 k} \int_{a}^{x}\left(1-e^{2 i k(x-t)}\right) q(t) v_{1}(t, k) d t \\
& v_{2}(x, k)=1+\frac{i}{2 k} \int_{x}^{b}\left(1-e^{2 i k(t-x)}\right) q(t) v_{2}(t, k) d t .
\end{aligned}
$$

При этом

$$
\begin{aligned}
y_{1}^{\prime}(a, k)-i k y_{1}(a, k) & =-2 i k e^{-i k a} \\
y_{2}^{\prime}(b, k)+i k y_{2}(b, k) & =2 i k e^{i k b}
\end{aligned}
$$

и, кроме того,

$$
\begin{aligned}
y_{1}^{\prime}(b, k)+i k y_{1}(b, k) & =e^{-i k b} v_{1}^{\prime}(b, k), \\
y_{2}^{\prime}(a, k)-i k y_{2}(a, k) & =e^{i k a} v_{2}^{\prime}(a, k) .
\end{aligned}
$$

Таким образом, вопрос об асимптотике собственных значений задачи (1)-(2) сводится к исследованию распределения корней уравнения

$$
e^{-2 i k(b-a)} v_{1}^{\prime}(b, k) v_{2}^{\prime}(a, k)=4 k^{2} .
$$

В $\S 2$ и 4 построены решения $v_{1}(x, k)$ и $v_{2}(x, k)$ указанных интегральных уравнений и изучено их асимптотическое поведение при больших по абсолютной величине значениях параметра $k \in \mathbb{C}_{+}$. В частности, там установлено, что

$$
\begin{aligned}
v_{1}^{\prime}(b, k) & =q_{0}(b)(b-a)^{\alpha} \Gamma(\beta+1) z^{-\beta-1}+\Phi_{1}(z)+\Psi_{1}(z), \\
-v_{2}^{\prime}(a, k) & =q_{0}(a)(b-a)^{\beta} \Gamma(\alpha+1) z^{-\alpha-1}+\Phi_{2}(z)+\Psi_{2}(z),
\end{aligned}
$$

где $z=-2 i k, \Phi_{1}(z)=O\left(z^{-\beta-2}\right), \Phi_{2}(z)=O\left(z^{-\alpha-2}\right), \Psi_{1,2}(z)=O\left(e^{-(b-a) \operatorname{Re} z}\right)$. Эти асимптотические формулы позволяют локализовать корни уравнения (6). Подстановка (7) и (8) в уравнение (6) приводит его после надлежащих преобразований к виду

$$
z-\lambda \ln z+\delta(z)=\omega_{n}, \quad n \in \mathbb{Z},
$$

где $\lambda:=\gamma /(b-a), \omega_{n}:=(2 \pi i n-\ln C) /(b-a)$ и $\delta(z)=O\left(z^{-1}\right)$. Известно (см., например, [10]), что трансцендентное уравнение

$$
z-\ln z=\omega
$$

при достаточно больших $\omega>0$ имеет единственное решение

$$
z(\omega)=\omega+\ln \omega+O\left(\frac{\ln \omega}{\omega}\right) .
$$

Аналогичное утверждение верно и для рассматриваемого здесь уравнения (9), а именно, при достаточно больших по абсолютной величине $n \in \mathbb{Z}$ оно имеет единственное решение

$$
z_{n}=\omega_{n}+\lambda \ln \omega_{n}+O\left(\frac{\ln |n|}{|n|}\right) .
$$


Проверка этого факта завершает доказательство основной теоремы, которому посвящен $\S 5$. В $\S 3$ установлены необходимые для дальнейшего асимптотические свойства интегралов типа Лапласа и развита техника асимптотических оценок, которая впоследствии применяется в 44 для получения формул (7) и (8). Всюду далее используются обозначения $\|f\|:=\sup _{x \in(a, b)}|f(x)|$ и $\langle x\rangle:=-[-x]$ для $x \in \mathbb{R}$.

\section{§ 2. Фундаментальная система решений уравнения (1)}

Пусть $p \in C^{1}(a, b)$ и $\int_{a}^{b}\left|p^{\prime}(t)\right| d t<\infty$. Введем обозначение

$$
D:=\{z=\sigma+i \tau|\sigma \geqslant 0,| z \mid \geqslant 4(b-a)\|p\|\}
$$

и рассмотрим для $z \in D$ интегральное уравнение

$$
u(x, z)=1+\frac{1}{z} \int_{a}^{x}\left(1-e^{-z(x-t)}\right) p(t) u(t, z) d t .
$$

УтВеРЖДЕНИЕ 1. При $z \in D$ уравнение (10) имеет решение

$$
u(x, z)=1+\frac{1}{z} \int_{a}^{x} p(t) d t+U(x, z),
$$

где $\|U(\cdot, z)\|=O\left(z^{-2}\right)$ и, кроме того, $\left\|u^{\prime}(\cdot, z)\right\| \leqslant 2(b-a)\|p\|$.

ДокАЗАТЕЛЬство. Зафиксируем $z \in D$ и рассмотрим интегральный оператор $T: C[a, b] \rightarrow C[a, b]$, соответствующий уравнению (10):

$$
(T \varphi)(x, z)=\frac{1}{z} \int_{a}^{x}\left(1-e^{-z(x-t)}\right) p(t) \varphi(t) d t .
$$

Поскольку $z \in D$, то имеет место неравенство

$$
\|T\| \leqslant \frac{2(b-a)\|p\|}{|z|} \leqslant \frac{1}{2} .
$$

Таким образом, оператор $T$ сжимающий и решение $u(x, z)$ уравнения $(10)$ может быть построено методом последовательных приближений в виде ряда

$$
u(x, z):=1+\sum_{n=1}^{\infty}\left(T^{n} 1\right)(x, z) .
$$

Из оценки нормы оператора $T$ следует, что

$$
\|u(\cdot, z)-1\| \leqslant \sum_{n=1}^{\infty}\|T\|^{n}=\frac{\|T\|}{1-\|T\|} \leqslant \frac{4(b-a)\|p\|}{|z|} \leqslant 1 .
$$

Перепишем уравнение (10) в форме

$$
u(x, z)=1+\frac{1}{z} \int_{a}^{x} p(t) d t+U(x, z),
$$


где

$$
\begin{gathered}
U(x, z)=\frac{1}{z^{2}}\left(e^{-z(x-a)} p(a)-p(x)+\int_{a}^{x} e^{-z(x-t)} p^{\prime}(t) d t\right) \\
+\frac{1}{z} \int_{a}^{x}\left(1-e^{-z(x-t)}\right) p(t)(u(t, z)-1) d t .
\end{gathered}
$$

Используя оценку нормы $\|u(\cdot, z)-1\|$, получаем

$$
\|U(\cdot, z)\| \leqslant \frac{1}{|z|^{2}}\left(2\|p\|\left(1+4(b-a)^{2}\|p\|\right)+\int_{a}^{b}\left|p^{\prime}(t)\right| d t\right) .
$$

Далее, поскольку $u(\cdot, z) \in C[a, b]$ при любом $z \in D$, то

$$
u^{\prime}(x, z)=\int_{a}^{x} e^{-z(x-t)} p(t) u(t, z) d t
$$

и, стало быть, $\left\|u^{\prime}(\cdot, z)\right\| \leqslant 2(b-a)\|p\|$ для $z \in D$. Таким образом, утверждение полностью доказано.

Пусть $v_{1}(x, k)$ и $v_{2}(x, k)$ - решения интегральных уравнений $(4)$ и $(5)$, первое из которых имеет вид (10), а второе может быть записано в следующем виде:

$$
v_{2}(a+b-y, k)=1+\frac{i}{2 k} \int_{a}^{y}\left(1-e^{2 i k(y-s)}\right) q(a+b-s) v_{2}(a+b-s, k) d s .
$$

Непосредственно проверяется, что

$$
y_{1}(x, k):=e^{-i k x} v_{1}(x, k), \quad y_{2}(x, k):=e^{i k x} v_{2}(x, k)
$$

удовлетворяют уравнению (1), причем вронскиан этих двух решений (в точке $x=b)$ имеет вид

$$
\left|\begin{array}{cc}
e^{-i k b} v_{1}(b, k) & e^{i k b} \\
e^{-i k b}\left[v_{1}^{\prime}(b, k)-i k v_{1}(b, k)\right] & i k e^{i k b}
\end{array}\right|=2 i k v_{1}(b, k)-v_{1}^{\prime}(b, k) .
$$

Из утверждения 1 следует, что для $k \in \mathbb{C}_{+}$при $|k| \rightarrow \infty$

$$
2 i k v_{1}(b, k)-v_{1}^{\prime}(b, k)=2 i k+O(1) \neq 0,
$$

и, таким образом, для $k \in \mathbb{C}_{+}$, достаточно больших по абсолютной величине, $\left\{y_{1}(x, k), y_{2}(x, k)\right\}-\Phi С Р$ уравнения $(1)$. В случае $k \in \mathbb{C}_{-}$в качестве ФСР уравнения (1) можно выбрать $\widetilde{y}_{1}(x, k)=y_{1}(x,-k)$ и $\widetilde{y}_{2}(x, k)=y_{2}(x,-k)$. Поскольку $v_{1,2}(x, \pm k)$ суть решения интегральных уравнений типа (10), то они, а следовательно и $y_{1,2}(x, \pm k)$, являются аналитическими функциями параметра $k$ при каждом фиксированном $x \in[a, b]$.

Пусть $\left\{\varphi_{1}(x, k), \varphi_{2}(x, k)\right\}-$ ФСР уравнения (1). Функция

$$
\Delta\left[\varphi_{1}, \varphi_{2}\right](k)=\left|\begin{array}{ll}
\varphi_{1}^{\prime}(a, k)-i k \varphi_{1}(a, k) & \varphi_{2}^{\prime}(a, k)-i k \varphi_{2}(a, k) \\
\varphi_{1}^{\prime}(b, k)+i k \varphi_{1}(b, k) & \varphi_{2}^{\prime}(b, k)+i k \varphi_{2}(b, k)
\end{array}\right|
$$


называется характеристическим определителем задачи (1)-(2). Предположим, что $\left\{\widetilde{\varphi}_{1}(x, k), \widetilde{\varphi}_{2}(x, k)\right\}$ - некоторая другая ФСР уравнения $(1)$ и $C(k)-$ матрица перехода от первой системы ко второй. Тогда

$$
\Delta\left[\widetilde{\varphi}_{1}, \widetilde{\varphi}_{2}\right](k)=\operatorname{det} C(k) \Delta\left[\varphi_{1}, \varphi_{2}\right](k)
$$

и, стало быть, нули характеристического определителя не зависят от выбора $\Phi \mathrm{CP}$, поскольку $\operatorname{det} C(k) \neq 0$. Если начальные данные $\varphi_{j}(a, k)$ и $\varphi_{j}^{\prime}(a, k)$, $j=1,2,-$ аналитические функции параметра $k$, то функции $\varphi_{j}(x, k)$ будут аналитическими при любом фиксированном $x \in[a, b]$ (см. [11]). Следовательно, характеристический определитель задачи (1)-(2), построенный по такой $\Phi С Р$, - аналитическая функция переменной $k$.

Известно (см. [9]), что спектр задачи (1)-(2) дискретный и совпадает с нулями ее характеристического определителя. Из вышесказанного вытекает, что достаточно большие по абсолютной величине собственные значения $k \in \mathbb{C}_{+}$ задачи (1)-(2) суть нули аналитической функции

$$
\Delta(k):=\Delta\left[y_{1}, y_{2}\right](k)=\left|\begin{array}{cc}
-2 i k e^{-i k a} & e^{i k a} v_{2}^{\prime}(a, k) \\
e^{-i k b} v_{1}^{\prime}(b, k) & 2 i k e^{i k b}
\end{array}\right| .
$$

В свою очередь точки спектра задачи (1)-(2) в $\mathbb{C}_{-}$являются нулями определителя

$$
\begin{aligned}
\widetilde{\Delta}(k) & :=\Delta\left[\widetilde{y_{1}}, \widetilde{y_{2}}\right](k) \\
& =\left|\begin{array}{cc}
0 & e^{-i k a}\left[v_{2}^{\prime}(a,-k)-2 i k v_{2}(a,-k)\right] \\
e^{i k b}\left[v_{1}^{\prime}(b,-k)+2 i k v_{1}(b,-k)\right] & 0
\end{array}\right| .
\end{aligned}
$$

В силу утверждения 1 для $k \in \mathbb{C}_{-},|k| \geqslant 2(b-a)\|q\|$, имеет место оценка

$$
\left|v_{1}(b,-k)+\frac{v_{1}^{\prime}(b,-k)}{2 i k}\right| \geqslant\left|v_{1}(b,-k)\right|-\frac{\left|v_{1}^{\prime}(b,-k)\right|}{2|k|} \geqslant 1-\frac{3(b-a)\|q\|}{|k|}
$$

и, аналогично,

$$
\left|v_{2}(a,-k)-\frac{v_{2}^{\prime}(a,-k)}{2 i k}\right| \geqslant 1-\frac{3(b-a)\|q\|}{|k|} .
$$

Отсюда следует, что при $|k|>3(b-a)\|q\|$ определитель $\widetilde{\Delta}(k)$ не обращается в нуль и, таким образом, установлено

ПРЕДЛОЖЕНИЕ 1. В нижней полуплоскости $\mathbb{C}_{-}$находится лищъ конечное число собственных значений задачи (1)-(2), причем все они расположены в круге $\{|k| \leqslant 3(b-a)\|q\|\}$.

\section{§ 3. Асимптотика интегралов типа Лапласа}

Введем обозначение $a_{\sigma}:=a+\sigma^{-1}$, где $\sigma>1 /(b-a)$ так, что $a_{\sigma} \in(a, b)$. Рассмотрим интеграл типа Лапласа

$$
I(f, z):=\int_{a_{\sigma}}^{b} e^{-z(b-x)} f(x) d x
$$

с комплексным параметром $z=\sigma+i \tau$ и изучим его асимптотическое поведение при $\sigma \rightarrow \infty$. Предварительно дадим следующее 
ОПРЕДЕЛЕниЕ 1 . Функция $f:(a, b) \rightarrow \mathbb{R}$ принадлежит классу $(\eta|N| \theta)$, где $\eta, \theta \in \mathbb{R}$ и $N \in \mathbb{N}$, если $f \in C^{N}(a, b)$ и для $\kappa \leqslant N$ и $\delta \in(a, b)$ найдется такое $c_{\kappa}^{\delta}>0$, что

$$
\left|f^{(\kappa)}(x)\right| \leqslant c_{\kappa}^{\delta} \begin{cases}{\left[1+(x-a)^{\eta-\kappa}\right],} & x \in(a, \delta] ; \\ (b-x)^{\theta-\kappa}, & x \in[\delta, b) .\end{cases}
$$

Перечислим ряд свойств функций $f \in(\eta|N| \theta)$, которые используются в дальнейшем. Отметим прежде всего, что $f^{\prime} \in(\eta-1|N-1| \theta-1)$, а при выполнении условия $\theta>-1$ имеет место включение

$$
f^{(-1)}(x):=-\int_{x}^{b} f(t) d t \in(\eta|N+1| \theta+1) .
$$

Если $g \in\left(\eta^{\prime}\left|N^{\prime}\right| \theta^{\prime}\right)$, то, как показывает непосредственная проверка,

$$
f(x) g(x) \in\left(\min \left\{\eta, \eta^{\prime}, \eta+\eta^{\prime}\right\}\left|\min \left\{N, N^{\prime}\right\}\right| \theta+\theta^{\prime}\right) .
$$

Лемма 1. При $N=\langle\theta\rangle u \theta>-1$ для $f \in(\eta|N| \theta)$ найдется $A>0$ такое, что интеграль

$$
\int_{a_{\sigma}}^{b}\left|e^{-z(b-x)} f^{(\kappa)}(x)\right| d x, \quad \kappa=0, \ldots, N,
$$

ограничены равномерно по $z=\sigma+i \tau$, если $\sigma \geqslant A$; кроме того, справедливо равенство

$$
I(f, z)=I_{1}(f, z)+I_{2}(f, z)
$$

¿əe

$$
I_{1}(f, z):=\frac{(-1)^{N}}{z^{N}} I\left(f^{(N)}, z\right)=O\left(z^{-N}\right), \quad I_{2}(f, z)=O\left(\left[1+\sigma^{-\eta}\right] e^{-\sigma(b-a)}\right) .
$$

ДокАЗАТЕЛЬСтво. Производная функции $f$ порядка $\kappa \leqslant N=\langle\theta\rangle$ имеет суммируемую особенность в точке $x=b$, поскольку $\left|f^{(\kappa)}(x)\right| \leqslant c_{\kappa}^{\delta}(b-x)^{\theta-\kappa}$. Зафиксируем $\delta \in(a, b)$ и положим

$$
S_{\kappa}^{\delta}(f):=\int_{\delta}^{b}\left|f^{(\kappa)}(x)\right| d x<\infty, \quad \kappa=0, \ldots, N .
$$

Ввиду оценки $\left|f^{(\kappa)}(x)\right| \leqslant c_{\kappa}^{\delta}\left[1+(x-a)^{\eta-\kappa}\right]$, выполненной при $x \in(a, \delta]$, имеем

$$
\begin{gathered}
\left(\int_{a_{\sigma}}^{\delta}+\int_{\delta}^{b}\right)\left|e^{-z(b-x)} f^{(\kappa)}(x)\right| d x \leqslant \int_{a_{\sigma}}^{\delta} e^{-\sigma(b-x)}\left|f^{(\kappa)}(x)\right| d x+S_{\kappa}^{\delta}(f) \\
\leqslant c_{\kappa}^{\delta}\left[b-a+\int_{a_{\sigma}}^{\delta} e^{-\sigma(b-x)}(x-a)^{\eta-\kappa} d x\right]+S_{\kappa}^{\delta}(f),
\end{gathered}
$$

где $a_{\sigma} \in(a, \delta]$, если $\sigma \geqslant 1 /(\delta-a)$, и, кроме того,

$$
\begin{array}{r}
\int_{a_{\sigma}}^{\delta} e^{-\sigma(b-x)}(x-a)^{\eta-\kappa} d x \leqslant e^{-\sigma(b-\delta)} \int_{a_{\sigma}}^{\delta}(x-a)^{\eta-\kappa} d x \\
=e^{-\sigma(b-\delta)} \begin{cases}\frac{(\delta-a)^{\eta-\kappa+1}-\sigma^{\kappa-\eta-1}}{\eta-\kappa+1}, & \eta \neq \kappa-1 \\
\ln \sigma(\delta-a), & \eta=\kappa-1 .\end{cases}
\end{array}
$$


Вследствие этих неравенств существуют $A \geqslant 1 /(\delta-a)$ и $\widetilde{C}>0$ такие, что

$$
\int_{a_{\sigma}}^{b}\left|e^{-z(b-x)} f^{(\kappa)}(x)\right| d x \leqslant \widetilde{C} \quad \text { при } \sigma \geqslant A, 0 \leqslant \kappa \leqslant N .
$$

Интегрируя $I(f, z)$ по частям $N$ раз и учитывая, что $f^{(\kappa)}(b)=0$ при $\kappa=$ $0, \ldots, N-1$, получаем

$$
\begin{aligned}
\int_{a_{\sigma}}^{b} e^{-z(b-x)} & f(x) d x=\frac{(-1)^{N}}{z^{N}} \int_{a_{\sigma}}^{b} e^{-z(b-x)} f^{(N)}(x) d x \\
& +\sum_{\kappa=1}^{N} \frac{(-1)^{\kappa}}{z^{\kappa}} f^{(\kappa-1)}\left(a_{\sigma}\right) e^{-z\left(b-a_{\sigma}\right)}=I_{1}(f, z)+I_{2}(f, z) .
\end{aligned}
$$

Согласно доказанному для $\sigma \geqslant A$ выполнена оценка

$$
\left|I_{1}(f, z)\right| \leqslant \frac{1}{|z|^{N}} \int_{a_{\sigma}}^{b}\left|e^{-z(b-x)} f^{(N)}(x)\right| d x \leqslant \frac{\widetilde{C}}{|z|^{N}} .
$$

Поскольку $f \in(\eta|N| \theta)$, то $\left|f^{(\kappa-1)}\left(a_{\sigma}\right)\right| \leqslant c_{\kappa-1}^{\delta}\left[1+\sigma^{\kappa-\eta-1}\right]$ при $1 \leqslant \kappa \leqslant N$ и, следовательно, для $\sigma \geqslant \max \{1, A\}$ справедливо неравенство

$$
\begin{aligned}
\left|I_{2}(f, z)\right| & \leqslant\left(\sum_{\kappa=1}^{N} \frac{c_{\kappa-1}^{\delta}}{|z|^{\kappa}}+\sigma^{-\eta} \sum_{\kappa=1}^{N} \frac{c_{\kappa-1}^{\delta} \sigma^{\kappa-1}}{|z|^{\kappa}}\right) e^{-\sigma\left(b-a_{\sigma}\right)} \\
& \leqslant\left[1+\sigma^{-\eta}\right] e^{-\sigma(b-a)+1} \sum_{\kappa=1}^{N} c_{\kappa-1}^{\delta} .
\end{aligned}
$$

Лемма доказана.

УтВеРЖДЕНИЕ 2. Пусть $g \in(\eta|N| \theta)$, где $N=\langle\theta\rangle+2 u \theta\rangle-1$, причем $g(x)(b-x)^{-\theta}=: g_{0}(x) \in C^{N}(a, b]$. Тогда при достаточно больших значениях $\sigma=\operatorname{Re} z$ имеет место равенство

$$
I(g, z)=g_{0}(b) \Gamma(\theta+1) z^{-\theta-1}+\widetilde{I}_{1}(g, z)+\widetilde{I}_{2}(g, z),
$$

в котором $\widetilde{I}_{1}(g, z)=O\left(z^{-\theta-2}\right)$ и $\widetilde{I}_{2}(g, z)=O\left(\left[1+\sigma^{-\eta}\right] e^{-\sigma(b-a)}\right)$.

ДокАЗАТЕЛЬство. Введем в рассмотрение функцию

$$
\Phi(x):=g_{0}(x)-g_{0}(b)-g_{0}^{\prime}(b)(x-b) .
$$

Поскольку $g \in(\eta|N| \theta)$, то $g_{0} \in(\eta|N| 0)$ и, стало быть, $\Phi \in(\eta|N| 0)$. С учетом интегрального представления

$$
\Phi(x)=\int_{x}^{b} d t \int_{t}^{b} g_{0}^{\prime \prime}(s) d s
$$

в котором $g_{0}^{\prime \prime} \in C^{N-2}(a, b]$ и $N-2=\langle\theta\rangle \geqslant 0$, заключаем, что $\Phi \in(\eta|N| 2)$. Далее, подставим выражение

$$
g(x)=g_{0}(b)(b-x)^{\theta}-g_{0}^{\prime}(b)(b-x)^{\theta+1}+r(x),
$$


где $r(x):=\Phi(x)(b-x)^{\theta} \in(\eta|N| \theta+2)$, в интеграл

$$
\begin{aligned}
I(g, z)=g_{0}(b) & \int_{a_{\sigma}}^{b} e^{-z(b-x)}(b-x)^{\theta} d x \\
& -g_{0}^{\prime}(b) \int_{a_{\sigma}}^{b} e^{-z(b-x)}(b-x)^{\theta+1} d x+I(r, z)
\end{aligned}
$$

и рассмотрим слагаемые в правой части равенства (14) по отдельности. Интеграл из первого слагаемого запишем в виде

$$
\int_{a_{\sigma}}^{b} e^{-z(b-x)}(b-x)^{\theta} d x=\int_{0}^{b-a_{\sigma}} e^{-z t} t^{\theta} d t=z^{-\theta-1} \Gamma(\theta+1)+R_{1}(z),
$$

где для остатка $R_{1}(z)$ при $\sigma \geqslant 1$ выполнена оценка

$$
\begin{aligned}
\left|R_{1}(z)\right| & \leqslant \int_{b-a_{\sigma}}^{\infty} e^{-\sigma t} t^{\theta} d t=e^{-\sigma\left(b-a_{\sigma}\right)} \int_{b-a_{\sigma}}^{\infty} e^{-\sigma\left(t-\left(b-a_{\sigma}\right)\right)} t^{\theta} d t \\
& \leqslant e^{-\sigma(b-a)+1} \int_{b-a_{\sigma}}^{\infty} e^{-\left(t-\left(b-a_{\sigma}\right)\right)} t^{\theta} d t \leqslant e^{1+(b-a)} \Gamma(\theta+1) e^{-\sigma(b-a)}
\end{aligned}
$$

Аналогично для интеграла из второго слагаемого в (14) получаем

$$
\int_{a_{\sigma}}^{b} e^{-z(b-x)}(b-x)^{\theta+1} d x=z^{-\theta-2} \Gamma(\theta+2)+R_{2}(z),
$$

причем

$$
\left|R_{2}(z)\right| \leqslant e^{1+(b-a)} \Gamma(\theta+2) e^{-\sigma(b-a)} .
$$

Наконец, поскольку $r \in(\eta|N| \theta+2)$, где $N=\langle\theta\rangle+2$, то вследствие леммы 1 существует $A \geqslant 1$ такое, что при $\sigma \geqslant A$ имеет место разложение

$$
I(r, z)=\int_{a_{\sigma}}^{b} e^{-z(b-t)} r(t) d t=I_{1}(r, z)+I_{2}(r, z),
$$

в котором

$$
I_{1}(r, z)=O\left(z^{-\langle\theta\rangle-2}\right), \quad I_{2}(r, z)=O\left(\left[1+\sigma^{-\eta}\right] e^{-\sigma(b-a)}\right) .
$$

Возвращаясь к равенству (14) и вводя обозначения

$$
\begin{aligned}
& \widetilde{I}_{1}(g, z)=-g_{0}^{\prime}(b) \Gamma(\theta+2) z^{-\theta-2}+I_{1}(r, z), \\
& \widetilde{I}_{2}(g, z)=g_{0}(b) R_{1}(z)-g_{0}^{\prime}(b) R_{2}(z)+I_{2}(r, z),
\end{aligned}
$$

получаем искомое представление (13) с указанными в формулировке утверждения оценками величин $\widetilde{I}_{1}(g, z)$ и $\widetilde{I}_{2}(g, z)$.

\section{§ 4. Асимптотика решения интегрального уравнения (10)}

Рассмотрим построенное в $\S 2$ решение $u(x, z)$ интегрального уравнения $(10)$, предполагая, что функция $p(x)$ имеет в точках $a$ и $b$ нули порядков $\mu>0$ и $\nu>0$ соответственно:

$$
p(x)=(x-a)^{\mu} p_{0}(x)(b-x)^{\nu},
$$


и, кроме того, $p_{0} \in C^{\widetilde{N}}[a, b], \widetilde{N} \geqslant 1$. Нашей целью здесь будет вычисление асимптотики производной

$$
u^{\prime}(b, z)=\int_{a}^{b} e^{-z(b-x)} p(x) u(x, z) d x
$$

при больших по абсолютной величине значениях параметра $z \in D$. В конце настоящего параграфа будет доказано следующее

УтВеРЖДЕНИЕ 3. Пусть $p_{0} \in C^{\langle\nu\rangle+2}[a, b]$. Тогда при $\sigma \rightarrow \infty$ справедлива асимптотическая формула

$$
u^{\prime}(b, z)=p_{0}(b)(b-a)^{\mu} \Gamma(\nu+1) z^{-\nu-1}+O\left(z^{-\nu-2}\right)+O\left(e^{-\sigma(b-a)}\right) .
$$

Эффективным приемом изучения асимптотического поведения интегралов Лапласа является формула интегрирования по частям (см. [10]); при этом вид главного члена асимптотики и оценки остатков зависят от степени гладкости подынтегральной функции и от порядков ее нулей в точках $a$ и $b$. Ниже формула интегрирования по частям модифицируется применительно к нашей ситуации (т.е. с учетом специфики функции $u(x, z)$, входящей в подынтегральное выражение). Предварительно введем в рассмотрение интегро-дифференциальную операцию

$$
l: f(x) \longmapsto-f^{\prime}(x)+p(x) f^{(-1)}(x) ;
$$

согласно указанным в $\S 3$ свойствам функций класса $(\eta|N| \theta)$, будем иметь

$$
l:(\eta|N| \theta) \rightarrow(\eta-1|N-1| \theta-1),
$$

если $\eta \leqslant \mu, N \leqslant \widetilde{N}$ и $\theta>-1$.

Лемма 2. Если функиия $f \in C^{1}(a, b)$ имеет интегрируемую особенность в точке $x=b$, то для произвольного отрезка $[c, d] \subset(a, b)$ верно равенство

$$
\begin{aligned}
\int_{c}^{d} e^{-z(b-x)} f(x) u(x, z) d x & \\
= & \left.\frac{1}{z}\left(f(x) u(x, z) e^{-z(b-x)}-f^{(-1)}(x) \int_{a}^{x} e^{-z(b-t)} p(t) u(t, z) d t\right)\right|_{c} ^{d} \\
& +\frac{1}{z} \int_{c}^{d} e^{-z(b-x)} l(f)(x) u(x, z) d x
\end{aligned}
$$

При условии, что функиия $l(f)$ (равно как и $f^{\prime}$ ) имеет интегрируемуюо особенность в точке а или b, формула (17) остается справедливой при с =а или $d=b$ соответственно.

ДокАзАтЕЛЬСтво. В случае, когда $[c, d] \subset(a, b)$, проинтегрируем по частям

$$
\begin{aligned}
\int_{c}^{d} f(x) u(x, z) d e^{-z(b-x)} & =\left.f(x) u(x, z) e^{-z(b-x)}\right|_{c} ^{d} \\
& -\int_{c}^{d} e^{-z(b-x)} f^{\prime}(x) u(x, z) d x-\int_{c}^{d} e^{-z(b-x)} f(x) u^{\prime}(x, z) d x .
\end{aligned}
$$


Подставляя в последнее слагаемое правой части этого выражения вместо производной $u^{\prime}(x, z)$ ее интегральное представление $(12)$ и учитывая существование первообразной $f^{(-1)}(x)$, получаем равенство

$$
\begin{aligned}
\int_{c}^{d} & e^{-z(b-x)} f(x) u^{\prime}(x, z) d x=\int_{c}^{d}\left(\int_{a}^{x} e^{-z(b-x+x-t)} p(t) u(t, z) d t\right) d f^{(-1)}(x) \\
& =\left.\left(f^{(-1)}(x) \int_{a}^{x} e^{-z(b-t)} p(t) u(t, z) d t\right)\right|_{c} ^{d}-\int_{c}^{d} e^{-z(b-x)} p(x) f^{(-1)}(x) u(x, z) d x
\end{aligned}
$$

завершающее доказательство. Если $f^{\prime}$ (или, что эквивалентно, $l(f)$ ) имеет интегрируемую особенность в точке $a$ или $b$, то предельным переходом получается формула (17) при $c=a$ или $d=b$ соответственно.

Воспользуемся формулой (17) для получения "грубой" оценки производной $u^{\prime}(b, z)$ решения уравнения (10), которая будет использоваться в доказательстве основной теоремы (см. §5). Предположим, что $p_{0} \in C^{\langle s\rangle}[a, b]$, где $s=\min \{\mu, \nu\}$. Тогда $l^{n}(p) \in C^{\langle s\rangle-n}(a, b)$ при $n \leqslant\langle s\rangle$ и, кроме того, $l^{n}(p)(a)=l^{n}(p)(b)=0$, если $n<s$, а функция $l^{\langle s\rangle}(p) \in C(a, b)$ имеет интегрируемые особенности в точках $a$ и $b$. Применяя с учетом этого формулу (17) к интегралу (15), будем иметь

$$
\int_{a}^{b} e^{-z(b-x)} p(x) u(x, z) d x=\frac{1}{z^{\langle s\rangle}} \int_{a}^{b} e^{-z(b-x)} l^{\langle s\rangle}(p)(x) u(x, z) d x .
$$

В силу абсолютной интегрируемости функции $l^{\langle s\rangle}(p)$ на отрезке $[a, b]$ и равномерной по $z \in D$ ограниченности нормы $\|u(\cdot, z)\|$ (см. доказательство утверждения 1) интеграл в правой части последнего равенства равномерно ограничен по $z \in D$. Тем самым установлена

Лемма 3. Пусть $p_{0} \in C^{\langle s\rangle}[a, b]$, где $s=\min \{\mu, \nu\}$. Тогда существует $B>0$ такое, что для $z \in D$ справедлива оценка

$$
\left|u^{\prime}(b, z)\right|=\left|\int_{a}^{b} e^{-z(b-x)} p(x) u(x, z) d x\right| \leqslant B|z|^{-\langle s\rangle} .
$$

В дальнейшем наряду с оценкой, полученной в лемме 3, нам понадобится асимптотика $u^{\prime}(b, z)$ при $\sigma \rightarrow \infty$, метод получения которой также основывается на применении формулы (17). При этом указанную формулу приходится применять столько раз, каков порядок нуля функции $p(x)$ в точке $b$. Поскольку $p(x)$ имеет в точках $a$ и $b$ нули, вообще говоря, различных (и притом не обязательно целых) порядков, то производные больших порядков функции $p(x)$ могут иметь особенности в точке $a$, а значит применение формулы (17) будет невозможным. Чтобы избежать этой трудности, разобьем интеграл (15) на слагаемые, разделив отрезок $[a, b]$ точкой $a_{\sigma}=a+\sigma^{-1}$ на две части, и будем рассматривать соответствующие им интегралы по отдельности. Введем обозначение

$$
R(p, z):=\int_{a}^{a_{\sigma}} e^{-z(b-x)} p(x) u(x, z) d x
$$

и отметим, что (в силу оценки $\|u(\cdot, z)\| \leqslant 2$, фактически полученной в ходе доказательства утверждения 1) при $z \in D$ и $\sigma \geqslant 1$ справедливо неравенство

$$
|R(p, z)| \leqslant \int_{a}^{a_{\sigma}} e^{-\sigma(b-x)}|p(x) u(x, z)| d x \leqslant 4\|p\| e^{-\sigma(b-a)} .
$$


Лемма 4. Пусть $f \in(\eta|\langle\theta\rangle| \theta)$, причем $0 \leqslant\langle\theta\rangle \leqslant \widetilde{N} u \eta \leqslant \mu$. Тогда

$$
J(f, z):=\int_{a_{\sigma}}^{b} e^{-z(b-x)} f(x) u(x, z) d x=J_{1}(f, z)+J_{2}(f, z),
$$

где $J_{1}(f, z)=O\left(z^{-\langle\theta\rangle}\right)$ и $J_{2}(f, z)=O\left(\left[1+\sigma^{-\eta}\right] e^{-\sigma(b-a)}\right), \sigma \rightarrow \infty$.

ДокАЗАтЕльство. Для $n \leqslant\langle\theta\rangle$ функции $l^{n}(f),\left[l^{n}(f)\right]^{(-1)}$ принадлежат классу $(\eta-n|\langle\theta\rangle-n| \theta-n)$ и, следовательно, при достаточно больших $\sigma>0$ выполнены оценки

$$
l^{n}(f)\left(a_{\sigma}\right)=O\left(\left[1+\sigma^{n-\eta}\right]\right), \quad\left[l^{n}(f)\right]^{(-1)}\left(a_{\sigma}\right)=O\left(\left[1+\sigma^{n-\eta}\right]\right) .
$$

Кроме того, $l^{n}(f)(b)=\left[l^{n}(f)\right]^{(-1)}(b)=0$, если $n<\langle\theta\rangle$, а функция $l^{\langle\theta\rangle}(f)$ имеет в точке $b$ интегрируемую особенность. Это (в силу леммы 2) позволяет $\langle\theta\rangle$ раз применить формулу (17) к интегралу

$$
\begin{aligned}
J(f, z)= & \frac{J\left(l^{\langle\theta\rangle}(f), z\right)}{z^{\langle\theta\rangle}}+\left(\sum_{n=0}^{\langle\theta\rangle-1} \frac{l^{n}(f)(x)}{z^{n+1}} u(x, z) e^{-z(b-x)}\right. \\
& \left.-\sum_{n=0}^{\langle\theta\rangle-1} \frac{\left[l^{n}(f)\right]^{(-1)}(x)}{z^{n+1}} \int_{a}^{x} e^{-z(b-t)} p(t) u(t, z) d t\right)\left.\right|_{a_{\sigma}} ^{b} \\
= & \frac{J\left(l^{\langle\theta\rangle}(f), z\right)}{z^{\langle\theta\rangle}}-\sum_{n=0}^{\langle\theta\rangle-1} \frac{l^{n}(f)\left(a_{\sigma}\right)}{z^{n+1}} u\left(a_{\sigma}, z\right) e^{-z\left(b-a_{\sigma}\right)} \\
& +\sum_{n=0}^{\langle\theta\rangle-1} \frac{\left[l^{n}(f)\right]^{(-1)}\left(a_{\sigma}\right)}{z^{n+1}} R(p, z) .
\end{aligned}
$$

Учитывая оценки (18) и (19), а также равномерную по $z \in D$ ограниченность нормы $\|u(\cdot, z)\|$, заключаем, что

$$
\begin{aligned}
J_{2}(f, z) & :=-\sum_{n=0}^{\langle\theta\rangle-1} \frac{l^{n}(f)\left(a_{\sigma}\right)}{z^{n+1}} u\left(a_{\sigma}, z\right) e^{-z\left(b-a_{\sigma}\right)}+\sum_{n=0}^{\langle\theta\rangle-1} \frac{\left[l^{n}(f)\right]^{(-1)}\left(a_{\sigma}\right)}{z^{n+1}} R(p, z) \\
& =O\left(\left[1+\sigma^{-\eta}\right] e^{-\sigma(b-a)}\right), \quad \sigma \rightarrow \infty .
\end{aligned}
$$

Далее, вследствие оценки $\|u(\cdot, z)\| \leqslant 2$ при $z \in D$ имеем неравенство

$$
\left|J\left(l^{\langle\theta\rangle}(f), z\right)\right| \leqslant 2 \int_{a_{\sigma}}^{b}\left|e^{-z(b-x)} l^{\langle\theta\rangle}(f)(x)\right| d x,
$$

где (согласно лемме 1) в силу включения $l^{\langle\theta\rangle}(f) \in(\eta-\langle\theta\rangle|0| \theta-\langle\theta\rangle)$ последний интеграл равномерно по $z=\sigma+i \tau$ ограничен, если $\sigma$ достаточно велико. Таким образом,

$$
J_{1}(f, z):=J\left(l^{\langle\theta\rangle}(f), z\right) z^{-\langle\theta\rangle}=O\left(z^{-\langle\theta\rangle}\right), \quad \sigma \rightarrow \infty .
$$

ДОКАЗАТЕЛЬСТВО УТВЕРЖДЕНИЯ 3 . Поскольку $p \in(\mu|\tilde{N}| \nu)$, где $\widetilde{N} \geqslant\langle\nu\rangle$ и $\mu>0$, то ввиду леммы 4 и оценки (18) достаточно проверить, что при больших 
значениях $\sigma>0$ справедливо равенство

$$
\begin{aligned}
z^{\langle\nu\rangle} J_{1}(p, z) & =J\left(l^{\langle\nu\rangle}(p), z\right) \\
& =p_{0}(b)(b-a)^{\mu} \Gamma(\nu+1) z^{-\nu+\langle\nu\rangle-1}+O\left(z^{-\nu+\langle\nu\rangle-2}\right)+O\left(\sigma^{\langle\nu\rangle} e^{-\sigma(b-a)}\right) .
\end{aligned}
$$

Предварительно (индукцией по $n$ ) покажем, что для $n \leqslant\langle\nu\rangle$ имеет место представление

$$
l^{n}(p)(x)=(x-a)^{\mu-n} p_{n}(x)(b-x)^{\nu-n}+\widetilde{p}_{n}(x),
$$

где $p_{n} \in C^{\widetilde{N}-n}[a, b], \widetilde{p}_{n} \in(\mu-n+1|\tilde{N}-n+1| \nu-n+2)$ и, кроме того,

$$
p_{n}(b)=\frac{\Gamma(\nu+1)}{\Gamma(\nu-n+1)}(b-a)^{n} p_{0}(b) .
$$

База индукции обеспечивается условием, согласно которому равенство (20) справедливо при $n=0$, причем $\widetilde{p}_{0} \equiv 0$. Предположим, что для некоторого $n<\langle\nu\rangle$ имеет место представление $(20)$. Тогда

$$
l^{n+1}(p)(x)=(x-a)^{\mu-n-1} p_{n+1}(x)(b-x)^{\nu-n-1}+\widetilde{p}_{n+1}(x),
$$

где

$$
p_{n+1}(x)=[(\nu-n)(x-a)-(\mu-n)(b-x)] p_{n}(x)-(x-a)(b-x) p_{n}^{\prime}(x)
$$

и

$$
\widetilde{p}_{n+1}(x)=-\widetilde{p}_{n}^{\prime}(x)+p(x)\left[l^{n}(p)\right]^{(-1)}(x) .
$$

Из индуктивного предположения и явного вида $p_{n+1}(x)$ вытекает, что $p_{n+1} \in$ $C^{\widetilde{N}-n-1}[a, b]$ и

$$
p_{n+1}(b)=(\nu-n)(b-a) p_{n}(b)=\frac{\Gamma(\nu+1)}{\Gamma(\nu-n)}(b-a)^{n+1} p_{0}(b) .
$$

Принадлежность $\widetilde{p}_{n+1} \in(\mu-n|\tilde{N}-n| \nu-n+1)$ следует из предположения индукции, согласно которому $\widetilde{p}_{n} \in(\mu-n+1|\widetilde{N}-n+1| \nu-n+2)$, и из включения $\left[l^{n}(p)\right]^{(-1)} \in(\mu-n|\widetilde{N}-n+1| \nu-n+1)$.

Таким образом, в силу доказанного функция $l^{\langle\nu\rangle}(p)$ представима в виде $(20)$ с $n=\langle\nu\rangle$, причем поскольку $\widetilde{N}=\langle\nu\rangle+2$, то $p_{\langle\nu\rangle} \in C^{2}[a, b]$ и $\widetilde{p}_{\langle\nu\rangle} \in(\mu-\langle\nu\rangle+1$ $|2| \theta+2)$, где $\theta=\nu-\langle\nu\rangle$ и, кроме того,

$$
p_{\langle\nu\rangle}(b)=\frac{\Gamma(\nu+1)}{\Gamma(\nu-\langle\nu\rangle+1)}(b-a)^{\langle\nu\rangle} p_{0}(b) .
$$

Положим

$$
g(x):=(x-a)^{\mu-\langle\nu\rangle} p_{\langle\nu\rangle}(x)(b-x)^{\nu-\langle\nu\rangle}, \quad \widetilde{g}(x):=g(x) \int_{a}^{x} p(t) d t .
$$

Используя формулы (20) и (11), представим интеграл $J\left(l^{\langle\nu\rangle}(p), z\right)$ в виде

$$
\begin{aligned}
& \int_{a_{\sigma}}^{b} e^{-z(b-x)} l^{\langle\nu\rangle}(p)(x) u(x, z) d x \\
& \quad=I(g, z)+\frac{I(\widetilde{g}, z)}{z}+\int_{a_{\sigma}}^{b} e^{-z(b-x)} g(x) U(x, z) d x+J\left(\widetilde{p}_{\langle\nu\rangle}, z\right)
\end{aligned}
$$


и оценим слагаемые этой суммы по отдельности. Из явного вида функции $g(x)$ и включения $p_{\langle\nu\rangle} \in C^{2}[a, b]$ следует, что $g \in(\mu-\langle\nu\rangle|2| \theta)$, где $-1<\theta \leqslant 0$, причем функция $g(x)(b-x)^{-\theta}$ дважды непрерывно дифференцируема на промежутке $(a, b]$. Таким образом, в силу утверждения 2 при $\sigma \rightarrow \infty$ имеем

$$
I(g, z)=\Gamma(\nu+1)(b-a)^{\mu} p_{0}(b) z^{-\nu+\langle\nu\rangle-1}+O\left(z^{-\nu+\langle\nu\rangle-2}\right)+O\left(\sigma^{\langle\nu\rangle} e^{-\sigma(b-a)}\right) .
$$

Далее, заметим, что $\widetilde{g}(x)=-p^{(-1)}(a) g(x)+p^{(-1)}(x) g(x)$, где $p^{(-1)} \in(\mu|\widetilde{N}| \nu+1)$, и, стало быть, $p^{(-1)} g \in(\mu-\langle\nu\rangle|1| \theta+1),\langle\theta+1\rangle=1$. Отсюда, используя утверждение 2 и лемму 1 , получаем

$$
I(\widetilde{g}, z)=O\left(z^{-\nu+\langle\nu\rangle-1}\right)+O\left(\sigma^{\langle\nu\rangle} e^{-\sigma(b-a)}\right), \quad \sigma \rightarrow \infty .
$$

Третье слагаемое в сумме (21) оценивается с помощью утверждения 1 и леммы 1. Действительно, поскольку $g \in(\mu-\langle\nu\rangle|0| \theta)$ и $\theta \in(-1,0]$, то

$$
\begin{gathered}
\left|\int_{a_{\sigma}}^{b} e^{-z(b-x)} g(x) U(x, z) d x\right| \leqslant\|U(\cdot, z)\| \int_{a_{\sigma}}^{b} e^{-\sigma(b-x)}|g(x)| d x=O\left(z^{-2}\right), \\
\sigma \rightarrow \infty
\end{gathered}
$$

Наконец, из включения $\widetilde{p}_{\langle\nu\rangle} \in(\mu-\langle\nu\rangle+1|2| \theta+2)$ ввиду леммы 4 вытекает асимптотическая при $\sigma \rightarrow \infty$ оценка

$$
\int_{a_{\sigma}}^{b} e^{-z(b-x)} \widetilde{p}_{\langle\nu\rangle}(x) u(x, z) d x=O\left(z^{-2}\right)+O\left(\sigma^{\langle\nu\rangle} e^{-\sigma(b-a)}\right),
$$

завершающая доказательство утверждения.

\section{§ 5. Локализация спектра задачи}

В § 2 показано, что спектр задачи (1)-(2) дискретный, т.е. состоит из изолированных собственных значений конечной кратности, совпадающих с нулями характеристического определителя, построенного по той или иной ФСР уравнения (1) (при этом совпадают также кратности собственного значения и соответствующего нуля характеристического определителя). Далее, там же установлено, что в нижней полуплоскости $\mathbb{C}_{-}$находится лишь конечное (возможно пустое) множество собственных значений.

Рассмотрим теперь часть спектра задачи (1)-(2), расположенную в верхней полуплоскости $\mathbb{C}_{+}$, и покажем, что искомые точки спектра - суть нули характеристического определителя $\Delta(k)$ - образуют две серии (простых, начиная с некоторого) собственных значений $k_{n}^{ \pm}$с асимптотиками вида (3). Предварительно выделим в $\mathbb{C}_{+}$зоны, свободные от точек спектра задачи (1)-(2).

Уравнение $\Delta(k)=0$ после замены $z=-2 i k$ принимает вид

$$
e^{z(b-a)} u_{1}^{\prime}(b, z) u_{2}^{\prime}(b, z)=z^{2},
$$

где $u_{1}(x, z)$ и $u_{2}(x, z)$ - решения интегральных уравнений

$$
u_{j}(x, z)=1+\frac{1}{z} \int_{a}^{x}\left(1-e^{-z(x-t)}\right) p_{j}(t) u_{j}(t, z) d t, \quad j=1,2,
$$


в которых $p_{1}(x)=q(x)$ и $p_{2}(x)=q(a+b-x)$, т.е. $v_{1}(x, k)=u_{1}(x,-2 i k)$ и $v_{2}(x, k)=u_{2}(a+b-x,-2 i k)$ представляют собой решения интегральных уравнений (4) и (5). Отметим, что при накладываемых на $q(x)$ ограничениях (cм. § 1$)$ функция $p_{1}(x)\left(p_{2}(x)\right)$ имеет в точках $a$ и $b$ нули порядков $\alpha$ и $\beta$ (соответственно $\beta$ и $\alpha)$, причем

$$
\begin{aligned}
& p_{1}(x)=(x-a)^{\alpha} q_{0}(x)(b-x)^{\beta}, \\
& p_{2}(x)=(x-a)^{\beta} q_{0}(a+b-x)(b-x)^{\alpha},
\end{aligned}
$$

где $q_{0}(a) q_{0}(b) \neq 0, q_{0} \in C^{m}[a, b]$ и $m \geqslant \varkappa:=\max \{\alpha, \beta\}+2$. Всюду ниже эти условия будут (по умолчанию) предполагаться выполненными.

ПРЕДЛОЖЕНИЕ 2. Существует $\sigma_{0}>0$ maкое, что множество

$$
\left\{z=\sigma+\left.i \tau\left|\sigma>\sigma_{0},\right| z\right|^{\varkappa} \geqslant e^{\sigma(b-a)}\right\}
$$

не содержит корней уравнения (22), а в полосе $\left\{0 \leqslant \sigma \leqslant \sigma_{0}\right\}$ находится лишь конечное их число.

ДокАЗАТЕЛьСтво. Без ограничения общности будем считать, что $\alpha \leqslant \beta$. Поскольку $m \geqslant \min \{\langle\alpha\rangle,\langle\beta\rangle\}$, то в силу леммы 3 найдется $B>0$ такое, что при $|z| \geqslant 4(b-a)\|q\|, \sigma \geqslant 0$, выполнены оценки

$$
\left|u_{j}^{\prime}(b, z)\right| \leqslant B|z|^{-\alpha}, \quad j=1,2 .
$$

Стало быть, если $z=\sigma+i \tau$ является решением уравнения (22) и $\sigma>\sigma_{1}:=$ $\max \left\{4(b-a)\|q\|, B^{2 / \alpha}\right\}$, то

$$
e^{-\sigma(b-a)}=\left|\frac{u_{1}^{\prime}(b, z) u_{2}^{\prime}(b, z)}{z^{2}}\right| \leqslant B^{2}|z|^{-2 \alpha-2}<|z|^{-\alpha-2}
$$

и, таким образом, множество $\left\{\sigma \geqslant \sigma_{1},|z|^{\alpha+2} \geqslant e^{\sigma(b-a)}\right\}$ не содержит корней уравнения (22).

Далее, ввиду условия $m \geqslant \max \{\langle\alpha\rangle,\langle\beta\rangle\}+2$ к $u_{j}^{\prime}(b, z)$ применимо утверждение 3 , согласно которому при $\sigma \rightarrow \infty$ справедливы асимптотические представления

$$
\begin{array}{ll}
u_{1}^{\prime}(b, z)=C_{1} z^{-\beta-1}+\Phi_{1}(z)+\Psi_{1}(z), & C_{1}=q_{0}(b)(b-a)^{\alpha} \Gamma(\beta+1), \\
u_{2}^{\prime}(b, z)=C_{2} z^{-\alpha-1}+\Phi_{2}(z)+\Psi_{2}(z), & C_{2}=q_{0}(a)(b-a)^{\beta} \Gamma(\alpha+1),
\end{array}
$$

где $\Phi_{1}(z)=O\left(z^{-\beta-2}\right), \Phi_{2}(z)=O\left(z^{-\alpha-2}\right), \Psi_{1,2}(z)=O\left(e^{-\sigma(b-a)}\right)$. Отсюда следует, что функция

$$
\psi(z):=\left(C_{1}+z^{\beta+1} \Phi_{1}(z)\right)\left[\frac{1}{C_{2}+z^{\alpha+1}\left(\Phi_{2}(z)+\Psi_{2}(z)\right)}-\frac{e^{z(b-a)} \Psi_{1}(z)}{z^{\alpha+3}}\right]^{-1}
$$

равномерно ограничена в области $\left\{|z|^{\alpha+2}<e^{\sigma(b-a)}\right\}$, если $\sigma$ достаточно велико. Выберем $\sigma_{0} \geqslant \sigma_{1}$ так, чтобы при $|z|^{\alpha+2}<e^{\sigma(b-a)}$ и $\sigma>\sigma_{0}$ выполнялось неравенство $|\psi(z)|<|z|^{\alpha+2}$. 
Пусть $z=\sigma+i \tau$ - решение уравнения (22) и $\sigma>\sigma_{0}$, тогда согласно доказанному $|z|^{\alpha+2}<e^{\sigma(b-a)}$. При этом соотношение (22) преобразуется к виду

$$
e^{-z(b-a)}=z^{-\alpha-\beta-4} \psi(z)
$$

откуда в силу выбора $\sigma_{0}$ получаем неравенство

$$
e^{-\sigma(b-a)}=|z|^{-\alpha-\beta-4}|\psi(z)|<|z|^{-\beta-2} .
$$

Тем самым установлено, что множество $\left\{\sigma>\sigma_{0},|z|^{\beta+2} \geqslant e^{\sigma(b-a)}\right\}$ не содержит корней уравнения (22).

Заметим наконец, что левая часть (22) в силу оценки (23) ограничена на множестве $\left\{0 \leqslant \sigma \leqslant \sigma_{0},|z| \geqslant 4(b-a)\|q\|\right\}$, в то время как правая часть там неограниченно возрастает с увеличением $|z|$. Следовательно, в полосе $\left\{0 \leqslant \sigma \leqslant \sigma_{0}\right\}$ находится лишь конечное число решений уравнения (22), и предложение полностью доказано.

Изучим теперь расположение корней уравнения (22) в области

$$
\Omega:=\left\{z=\sigma+\left.i \tau\left|\sigma>\sigma_{0},\right| z\right|^{\varkappa}<e^{\sigma(b-a)}\right\} .
$$

Введем в рассмотрение аналитическую в полуплоскости $\sigma>\sigma_{0}$ функцию

$$
\varphi(z):=\left(C_{1} C_{2}\right)^{-1} z^{\alpha+\beta+2} u_{1}^{\prime}(b, z) u_{2}^{\prime}(b, z),
$$

имеющую (вследствие утверждения 3) асимптотику $\varphi(z)=1+O\left(z^{-1}\right)$ при $|z| \rightarrow \infty, z \in \Omega$. Увеличим $\sigma_{0}$ так, чтобы при $z \in \Omega$ было выполнено неравенство $|\varphi(z)-1|<1$. Положим $C=C_{1} C_{2}$ и $\gamma=\alpha+\beta+4$ и перепишем уравнение $(22)$ в эквивалентной форме

$$
\exp (z(b-a)-\gamma \ln z+\ln C+\ln \varphi(z))=1 .
$$

Таким образом, $z \in \Omega$ является корнем уравнения (22) в том и только том случае, если для некоторого $n \in \mathbb{Z}$ имеет место равенство (9):

$$
z-\lambda \ln z+\delta(z)=\omega_{n}
$$

где $\lambda=\gamma /(b-a), \omega_{n}=(2 \pi i n-\ln C) /(b-a)$ и, кроме того, $\delta(z):=(\ln \varphi(z)) /(b-a)=$ $O\left(z^{-1}\right)$ при $|z| \rightarrow \infty, z \in \Omega$. Поскольку левая часть равенства (9) аналитична в полуплоскости $\sigma>\sigma_{0}$ и неограниченно возрастает с ростом модуля $z \in \Omega$, то (9) при любом фиксированном $n$ может иметь в $\Omega$ лишь конечное число решений. Доказательство основной теоремы, сформулированной в 1 , завершает

Лемма 5. Существуют $K>0$ и $M>0$ такие, что для каждого $n \in \mathbb{Z}$, $|n| \geqslant M$, уравнение (9) имеет в $\Omega$ единственный корень $z_{n}$, причем

$$
\left|z_{n}-\omega_{n}-\lambda \ln \omega_{n}\right| \leqslant K \frac{\ln |n|}{|n|} .
$$

ДокАЗАТЕЛЬство. Поскольку функция $z-\lambda \ln z+\delta(z)$ ограничена на любом компакте в $\Omega$, а $\left|\omega_{n}\right| \rightarrow \infty$ при $|n| \rightarrow \infty$, то для любого $R>0$ существует 
$N(R)>0$ такое, что множество $\Omega \cap\{|z| \leqslant R\}$ не содержит корней (9), если $|n| \geqslant N(R)$. После деления на z уравнение (9) принимает вид

$$
1-\frac{\omega_{n}}{z}=\lambda \frac{\ln z}{z}-\frac{\delta(z)}{z},
$$

где правая часть стремится к нулю, когда $|z| \rightarrow \infty, z \in \Omega$. Поэтому для произвольного $r>0$ найдется $R(r)>1 / r$ такое, что множество $\left\{z \in \Omega,\left|z / \omega_{n}-1\right| \geqslant r\right.$, $|z|>R(r)\}$ также не содержит решений уравнения (9). Стало быть, корни уравнения (9) при $|n| \geqslant N(R(r))$ могут находиться только в областях

$$
\Omega_{n}(r):=\left\{z \in \Omega,\left|\frac{z}{\omega_{n}}-1\right|<r,|z|>R(r)\right\} .
$$

Выберем и зафиксируем теперь $\rho>0$ так, чтобы для $z \in \Omega_{n}(\rho)$ выполнялось неравенство $\left|\lambda \ln \left(z / \omega_{n}\right)\right|+|\delta(z)| \leqslant 1 / 2$. Замена $z(\zeta)=\omega_{n}+\lambda \ln \omega_{n}+\zeta$ приводит (9) к виду

$$
\zeta=\lambda \ln \left(\frac{z(\zeta)}{\omega_{n}}\right)-\delta(z(\zeta))
$$

В силу выбора $\rho$ множество $\left\{|\zeta| \geqslant 1, z(\zeta) \in \Omega_{n}(\rho)\right\}$ не содержит корней уравнения (24). Далее, если $|\zeta| \leqslant 1$, то для достаточно больших $|n|$ имеют место двусторонние оценки

$$
|z(\zeta)| \asymp|n|, \quad \operatorname{Re} z(\zeta) \asymp \lambda \ln |n|, \quad e^{\operatorname{Re} z(\zeta)(b-a)} \asymp|n|^{\gamma} .
$$

Стало быть, существует $M \geqslant N(R(\rho))$ такое, что при $|n| \geqslant M$ и $|\zeta| \leqslant 1$ справедливы неравенства

$$
\left|\frac{z(\zeta)}{\omega_{n}}-1\right|<\rho, \quad|z(\zeta)|>R(\rho)
$$

и, кроме того,

$$
\operatorname{Re} z(\zeta)>\sigma_{0}, \quad e^{\operatorname{Re} z(\zeta)(b-a)}>|z(\zeta)|^{\varkappa} .
$$

Другими словами, $z(\zeta) \in \Omega_{n}(\rho)$, если $|\zeta| \leqslant 1$ и $|n| \geqslant M$. Следовательно, в силу выбора $\rho$ на окружности $|\zeta|=1$ при $|n| \geqslant M$ имеет место неравенство

$$
|\zeta|>\left|\lambda \ln \left(\frac{z(\zeta)}{\omega_{n}}\right)-\delta(z(\zeta))\right|
$$

Согласно теореме Руше уравнение (24) при $n \geqslant M$ имеет ровно один (простой) корень $\zeta_{n}$ в круге $|\zeta| \leqslant 1$. Более того, в силу равенства $(24)$ и оценки $\delta(z)=O\left(z^{-1}\right)$ существует такое $K>0$, что

$$
\left|\zeta_{n}\right| \leqslant \max _{|\zeta| \leqslant 1}\left|\lambda \ln \left(1+\frac{\lambda \ln \omega_{n}}{\omega_{n}}+\frac{\zeta}{\omega_{n}}\right)+\delta(z(\zeta))\right| \leqslant K \frac{\ln |n|}{|n|} .
$$

Таким образом установлено, что при фиксированном $n \in \mathbb{Z},|n| \geqslant M$, уравнение (9) имеет в $\Omega$ единственный корень

$$
z_{n}:=z\left(\zeta_{n}\right)=\omega_{n}+\lambda \ln \omega_{n}+O\left(\frac{\ln |n|}{|n|}\right) .
$$




\section{Список литературы}

[1] В. де Альфаро, Т. Редже, Потенииальное рассеяние, Мир, М., 1966; пер. с англ.: V. de Alfaro, T. Regge, Potential scattering, North-Holland, Amsterdam; Wiley, New York, 1965.

[2] А.И. Базь, Я.Б. Зельдович, А. М. Переломов, Рассеяние, реакиии и распады в нерелятивиской квантовой механике, Наука, М., 1971.

[3] М. Рид, Б. Саймон, Методы современной математической физики. т. 4. Анализ операторов, Мир, М., 1982; пер. с англ.: M. Reed, B. Simon, Methods of modern mathematical physics. IV. Analysis of operators, Academic Press, New York-London, 1978.

[4] R. B. Melrose, Geometric scattering theory, Stanford Lectures, Cambridge Univ. Press, Cambridge, 1995.

[5] M. Zworski, "Counting scattering poles", Spectral and scattering theory, Proceedings of the Taniguchi international workshop (Sanda, Japan, 1992), Lecture Notes in Pure and Appl. Math., 161, Dekker, New York, 1994, 301-331.

[6] J. Sjöstrand, "A trace formula and review of some estimates for resonances", $M i$ crolocal analysis and spectral theory (Lucca, Italy, 1996), NATO Adv. Sci. Inst. Ser. C Math. Phys. Sci., 490, Kluwer Acad. Publ., Dordrecht, 1997, 377-437.

[7] M. Zworski, "Distribution of poles for scattering on the real line", J. Funct. Anal., 73:2 (1987), 277-296.

[8] М. В. Федорюк, Асимптотические методы для линейных обыкновенных дифференциальных уравнений, Наука, М., 1983; англ. пер.: M. V. Fedoryuk, Asymptotic analysis: linear ordinary differential equations, Springer-Verlag, Berlin, 1993.

[9] М. А. Наймарк, Линейные дифференциальные операторы, 2 изд., Наука, М., 1969; англ. пер. 1-го изд.: М. А. Naimark, Linear differential operators. I, II, Ungar Publ., New York, 1967-1968.

[10] М. В. Федорюк, Асимптотика: интеграль и ряды, Наука, М., 1987.

[11] В. Вазов, Асимптотические разложения решения обыкновенных дифференциальных уравнений, Мир, М., 1968; пер. с англ.: W. Wasow, "Asymptotic expansions for ordinary differential equations", Pure Appl. Math., 14, Wiley, New York-LondonSydney, 1965.

\section{C. А. Степин (S. A. Stepin)}

Московский государственный

университет им. М. В. Ломоносова, механико-математический факультет

\section{А. Г. Тарасов (A. G. Tarasov)}

Московский государственный университет им. М. В. Ломоносова, механико-математический факультет E-mail: ag_tarasov@mail.ru 